\title{
Association of CA 15-3 and CEA with clinicopathological parameters in patients with metastatic breast cancer
}

\author{
BIAO GENG ${ }^{1}$, MAN-MAN LIANG ${ }^{2}$, XIAO-BING YE $^{1}$ and WEN-YING ZHAO ${ }^{1}$ \\ Departments of ${ }^{1}$ Medical Oncology and ${ }^{2}$ Internal Medicine, The First Affiliated Hospital \\ of Wannan Medical College, Wuhu, Anhui 241001, P.R. China
}

Received July 31, 2014; Accepted September 4, 2014

DOI: $10.3892 / \mathrm{mco} .2014 .419$

\begin{abstract}
The objective of this study was to investigate the association of serum cancer antigen 15-3 (CA 15-3) and carcinoembryonic antigen (CEA) levels with clinicopathological parameters in patients diagnosed with metastatic breast cancer (MBC). We retrospectively evaluated the medical records of 284 patients diagnosed with $\mathrm{MBC}$ between January, 2007 and December, 2012 who fulfilled the specified criteria and the association between the levels of the two tumor marker and clinicopathological parameters was analyzed. Of the 284 patients, elevated CA 15-3 and CEA levels at initial diagnosis of recurrence were identified in $163(57.4 \%)$ and 97 (34.2\%) patients, respectively. Elevated CA 15-3 and CEA levels were significantly associated with breast cancer molecular subtypes $(\mathrm{P}<0.001$ and $\mathrm{P}=0.032$, respectively). Cases with luminal subtypes exhibited a higher percentage of elevated CA 15-3 and CEA levels compared to non-luminal subtypes. Elevated CA 15-3 level was correlated with bone metastasis $(\mathrm{P}=0.017)$. However, elevation of $\mathrm{CEA}$ was observed regardless of the site of metastasis. Elevation of CA 15-3 was significantly more common in MBC with multiple metastatic sites compared to $\mathrm{MBC}$ with a single metastasis $(\mathrm{P}=0.001)$. However, the incidence of elevated CEA levels did not differ between patients with a single and those with multiple metastatic sites. In conclusion, elevated CA 15-3 and CEA levels at initial diagnosis of recurrence were found to be associated with breast cancer molecular subtypes, whereas an elevated CA 15-3 level was significantly correlated with bone metastasis and an elevated CEA level was observed regardless of metastatic site. The proportion of MBC cases with elevated CA 15-3 levels differed according to the number of metastatic sites.
\end{abstract}

Correspondence to: Dr Wen-Ying Zhao, Department of Medical Oncology, The First Affiliated Hospital of Wannan Medical College, 2 Zheshan West Road, Wuhu, Anhui 241001, P.R. China

E-mail: wnyxy3@163.com

Key words: metastatic breast cancer, cancer antigen 15-3, carcinoembryonic antigen, clinicopathological parameters

\section{Introduction}

Breast cancer is one of the most frequently occurring malignancies in China, with a steadily increasing incidence (1). Although the development of comprehensive therapy has reduced the mortality rate of breast cancer patients (2), regional and distant recurrences remain a major threat to breast cancer patients $(3,4)$. Serum tumor markers have been widely used as non-invasive tools for measuring treatment response, early diagnosis of recurrence and predicting prognosis. In breast cancer, the most widely used serum tumor markers are cancer antigen 15-3 (CA 15-3) and carcinoembryonic antigen (CEA). Although CA 15-3 and CEA are not currently recommended as markers for breast cancer screening or therapeutic response monitoring according to the American Society of Clinical Oncology guidelines (5), CA 15-3 and CEA are the markers most widely used for surveillance purposes and monitoring of treatment response in clinical practice.

Previous studies have suggested that elevated serum levels of CA 15-3 and CEA preoperatively were significantly associated with tumor size, axillary node metastasis and advanced stage in the multivariate analysis; in addition, patients with elevated CA 15-3 and CEA levels exhibited a worse prognosis compared to those with normal levels of CA 15-3 and CEA, even in stage-matched analysis $(6,7)$. Recent studies demonstrated that serial determination of CA 15-3 and CEA levels during the postoperative follow-up of breast cancer patients may be useful for early detection of preclinical recurrence or metastatic disease $(8,9)$. In addition, the serum levels of CA 15-3 and CEA may be used to predict response to chemotherapy in patients with metastatic breast cancer (MBC) (10). Similarly, a model for predicting disease progression using CA 15-3 and CEA in MBC patients without target lesions in first-line chemotherapy was suggested by Hashimoto et al (11), which delineated that evaluations of CA 15-3 and CEA at baseline contributed more significantly to the prediction of disease progression compared to other variables.

Despite continuous interest in this topic, only a limited number of studies have described the correlation of increased CA 15-3 and CEA levels at recurrence with clinicopathological parameters and specific sites of recurrence. A better understanding of the correlation between serum CA 15-3 and CEA levels and the intrinsic biological characteristics of breast cancer may affect the selection of adjuvant therapy and 
surveillance decisions and determine which investigations and therapies are appropriate upon diagnosis of distant disease.

In the present retrospective study, we analysed the association of serum CA 15-3 and CEA levels with clinicopathological parameters in patients with $\mathrm{MBC}$ and determined whether the elevation of these two tumor markers is correlated with metastatic site(s).

\section{Patients and methods}

Patient selection. This study was reviewed and approved by the Institutional Review Board and Academic Committee of The First Affiliated Hospital of Wannan Medical College, Wuhu, Anhui, China. Eligible subjects were selected among breast cancer patients with distant metastases diagnosed between January, 2007 and December, 2012 at the Department of Medical Oncology. The inclusion criteria were as follows: i) Data on serum tumor markers CA 15-3 and CEA were available when patients were diagnosed with $\mathrm{MBC}$; ii) complete patient clinicopathological and demographic data, including age at diagnosis, tumor histology, biological marker status, estrogen receptor (ER), progesterone receptor (PR) and human epidermal growth factor receptor 2 (HER2) status; Ki-67 proliferation index; staging information and grade; and anatomical site of first distant metastasis. All the patients were staged according to the American Joint Committee on Cancer (AJCC, 7th edition) TNM staging system for breast cancer. Distant metastasis refers to the presence of breast cancer lesions at sites distant to the primary site. The sites of distant relapse were categorized as follows: bone, brain, liver, lungs, distant lymph nodes and pleura.

Tumor marker analysis. The concentration of serum CA 15-3 and CEA levels were measured using chemiluminescent enzyme immunoassays (ADVIA Centaur; Siemens Medical Solutions Diagnostics, Tarrytown, NY, USA). The upper limits of normal for CA $15-3$ and CEA were $31.3 \mathrm{U} / \mathrm{ml}$ and $5 \mathrm{ng} / \mathrm{ml}$, respectively.

Immunohistochemistry. All the pathological specimens were reviewed by two experienced pathologists. Immunohistochemical staining was performed for ER, PR, HER2 and Ki-67. ER and PR positivity were defined as any positive nuclear staining $\geq 10 \%$. HER 2 was determined initially by IHC and graded between 0 and 3+. HER2-negative staining was defined as HER2 graded 0 or 1+; positive, as 3+; HER2 scores of $2+$ were determined as HER2-negative or -positive according to the results of fluorescence in situ hybridization analysis.

In this study, breast cancer was classified into 5 subtypes based on the expression of ER, PR, HER2 and Ki-67 proliferation index as follows: Luminal A, ER- and/or PR-positive and $\mathrm{Ki}-67<14 \%$; luminal B, ER- and/or PR-positive and Ki-67 >14\%; luminal/HER2: ER- and/or PR-positive and HER2-positive; HER2-enriched, ER- and PR-negative and HER2-positive; and triple-negative (TN) subtype: ER-, PRand HER2-negative.

Statistical analysis. The percentages of patients with elevated CA 15-3 and CEA levels were compared across
Table I. Patient characteristics.

\begin{tabular}{lrr}
\hline Characteristics & Patient no. $(\mathrm{n}=284)$ & $\%$ \\
\hline Patients with a & & \\
single metastasis & & \\
Bone & 66 & 23.2 \\
Brain & 5 & 1.8 \\
Liver & 14 & 4.9 \\
Lung & 28 & 9.9 \\
Distant nodal & 16 & 5.6 \\
Pleural & 7 & 2.5 \\
No. of metastatic sites & & \\
Single & 136 & 57.9 \\
Multiple & 148 & 52.1 \\
\hline
\end{tabular}

clinicopathological parameters using the Chi-square test or the Fisher's exact test, as appropriate; similar statistical tests were performed to compare the percentages of patients with CA 15-3 and CEA elevation by the first site(s) of metastasis or the number of metastatic sites. All the statistical assessments were two-sided and statistical significance was set at $\mathrm{P}<0.05$. Statistical analyses were performed with SPSS 17.0 software (SPSS, Inc., Chicago, IL, USA).

\section{Results}

Patient characteristics. The clinicopathological characteristics of $284 \mathrm{MBC}$ patients are summarized in Table I. The median age of the subjects was 48 years (range, 31-72 years). A total of 136 patients had a single metastatic site and 148 patients exhibited multiple metastatic sites. Patients with a single metastatic site were distributed as follows: 66 , bone $(23.2 \%)$; 5 , brain $(1.8 \%)$; 14 , liver $(4.9 \%) ; 28$, lung $(9.9 \%)$; 16 , distant nodes $(5.6 \%)$; and 7 , pleura $(2.5 \%)$.

Incidence of CA 15-3 and CEA elevation based on clinicopathological characteristics. Elevated CA 15-3 and CEA levels at initial diagnosis of recurrence were identified in $163(57.4 \%)$ and $97(34.2 \%)$ patients, respectively. Table II shows the correlation between serum CA 15-3 and CEA levels at initial diagnosis of recurrence and clinicopathological characteristics at the time of diagnosis of primary locoregional breast cancer (except for age and menopausal status, which were recorded at the time of first recurrence). Elevation of CA 15-3 and CEA levels at recurrence were only correlated with breast cancer molecular subtypes $(\mathrm{P}<0.001$ and $\mathrm{P}=0.032$, respectively). Age, menopausal status, tumor size, nodal status, TNM stage and histology, however, were not associated with an increase in the serum levels of these tumor markers. The increase in CA 15-3 levels according to breast cancer subtypes was as follows: Luminal A, 65.9\% (58/88); luminal B, 68.6\% (59/86); luminal/HER2, 62.2\% (23/37); HER2-enriched, $28.0 \%$ (7/25); and TN, 33.3\% (16/48). An elevated CA 15-3 level was most commonly observed in the luminal subtypes and the incidence of CA 15-3 elevation was lower in the HER2-enriched and $\mathrm{TN}$ subtypes. CEA elevation was observed in $35.1-42.0 \%$ of 
Table II. Correlation between serum CA 15-3 and CEA levels at initial diagnosis of recurrence and clinicopathological characteristics.

\begin{tabular}{|c|c|c|c|c|c|c|}
\hline \multirow[b]{2}{*}{ Characteristics } & \multicolumn{3}{|c|}{ CA 15-3 levels } & \multicolumn{3}{|c|}{ CEA levels } \\
\hline & $\begin{array}{l}\text { Normal }(\%) \\
\quad(n=121)\end{array}$ & $\begin{array}{l}\text { Elevated }(\%) \\
\quad(n=163)\end{array}$ & P-value & $\begin{array}{c}\text { Normal }(\%) \\
\quad(n=187)\end{array}$ & $\begin{array}{c}\text { Elevated }(\%) \\
\quad(n=97)\end{array}$ & P-value \\
\hline \multicolumn{7}{|l|}{ Age, years } \\
\hline$<40$ & $13(35.1)$ & $24(64.9)$ & \multirow[t]{2}{*}{0.324} & $21(56.8)$ & $16(43.2)$ & \multirow[t]{2}{*}{0.211} \\
\hline$\geq 40$ & $108(43.7)$ & $139(56.3)$ & & $166(67.2)$ & $81(32.8)$ & \\
\hline \multicolumn{7}{|l|}{ Menopausal status } \\
\hline Premenopausal & $57(46.7)$ & $65(53.3)$ & \multirow[t]{2}{*}{0.224} & $86(70.5)$ & $36(29.5)$ & \multirow[t]{2}{*}{0.152} \\
\hline Postmenopausal & $64(39.5)$ & $98(60.5)$ & & $101(62.3)$ & $61(37.7)$ & \\
\hline \multicolumn{7}{|l|}{ Tumor size } \\
\hline $\mathrm{T} 1$ & 39 (45.9) & $46(54.1)$ & \multirow[t]{2}{*}{0.466} & $62(72.9)$ & $23(27.1)$ & \multirow[t]{2}{*}{0.099} \\
\hline$\geq \mathrm{T} 2$ & $82(41.2)$ & $117(58.8)$ & & $125(62.8)$ & $74(37.2)$ & \\
\hline \multicolumn{7}{|l|}{ Nodal status } \\
\hline N0 & $54(38.3)$ & $87(61.7)$ & \multirow[t]{2}{*}{0.145} & $96(68.1)$ & 45 (31.9) & \multirow[t]{2}{*}{0.429} \\
\hline$\geq \mathrm{N} 1$ & $67(46.9)$ & $76(53.1)$ & & $91(63.6)$ & $52(36.4)$ & \\
\hline \multicolumn{7}{|l|}{ TNM stage } \\
\hline $\mathrm{I}$ & $27(50.9)$ & $26(49.1)$ & \multirow[t]{3}{*}{0.253} & $40(75.5)$ & $13(24.5)$ & \multirow[t]{3}{*}{0.166} \\
\hline II & $68(42.8)$ & $91(57.2)$ & & 98 (61.6) & $61(38.4)$ & \\
\hline III & $26(36.1)$ & $46(63.9)$ & & $49(68.1)$ & $23(31.9)$ & \\
\hline \multicolumn{7}{|l|}{ Histological grade } \\
\hline $\mathrm{I}$ & $32(45.1)$ & $39(54.9)$ & \multirow[t]{3}{*}{0.101} & $50(70.4)$ & $21(29.6)$ & \multirow[t]{3}{*}{0.607} \\
\hline II & $31(33.7)$ & $61(66.3)$ & & $58(63.0)$ & $34(37.0)$ & \\
\hline III & $58(47.9)$ & $63(52.1)$ & & $79(65.3)$ & $42(34.7)$ & \\
\hline \multicolumn{7}{|l|}{ Molecular subtype } \\
\hline Luminal A & $30(34.1)$ & $58(65.9)$ & \multirow[t]{5}{*}{$<0.001$} & $51(58.0)$ & $37(42.0)$ & \multirow[t]{5}{*}{0.032} \\
\hline Luminal B & $27(31.4)$ & $59(68.6)$ & & $53(61.6)$ & $33(38.4)$ & \\
\hline Luminal/HER2 & $14(37.8)$ & $23(62.2)$ & & $24(64.9)$ & $13(35.1)$ & \\
\hline HER2-enriched & $18(72.0)$ & $7(28.0)$ & & $21(84.0)$ & $4(16.0)$ & \\
\hline $\mathrm{TN}$ & $32(66.7)$ & $16(33.3)$ & & $38(79.2)$ & $10(20.8)$ & \\
\hline
\end{tabular}

CA 15-3, cancer antigen 15-3; CEA, carcinoembryonic antigen; HER2, human epidermal growth factor receptor 2; TN, triple-negative.

the luminal subtypes compared to only $16.0 \%(4 / 25)$ in the HER2-enriched type and $20.8 \%(10 / 48)$ in TN cases $(\mathrm{P}=0.032)$.

CA 15-3 and CEA elevation according to site(s) of first distant metastasis. We further investigated the association between elevated CA 15-3 and CEA levels and specific site of first distant metastasis. A total of 136 patients presented with a single metastatic site. Of the 136 patients, elevated CA $15-3$ and CEA levels at initial diagnosis of recurrence were identified in $64(47.1 \%)$ and $45(33.1 \%)$ patients, respectively. Among the patients with a single metastasis to the bone, liver, lung, distant nodes or pleura, an increased CA 15-3 level at recurrence was correlated with bone metastasis $(\mathrm{P}=0.017)$. However, there was no significant association between CEA elevation and specific site of distant metastasis (Table III). The increase in serum CA 15-3 differed based on the number of metastatic sites. Among patients with multiple metastatic sites, the proportion of patients with an increased CA 15-3 level was 66.9\%, which was statistically significantly different from the proportion of patients presenting with a single metastasis $(47.1 \% ; \mathrm{P}=0.001)$. However, the incidence of elevated CEA levels did not differ significantly between patients with a single and those with multiple metastatic sites (Table IV).

\section{Discussion}

Breast cancer is a heterogeneous group of diseases that differ in their pathological and molecular characteristics and clinical behavior. Despite advances in treatment, $20-30 \%$ of patients with early breast cancer eventually develop relapse with distant metastatic disease (2). The risk of recurrence is affected by stage at initial presentation and the underlying biology of the tumor. To improve early detection of recurrence and survival, CA 15-3 and CEA are widely used for the early diagnosis of metastatic disease in the clinical setting. During follow-up, CA 15-3 and CEA levels have been shown to detect 40-60\% 
Table III. Correlation between CA 15-3 and CEA elevation and site of first distant metastasis in patients with single metastasis.

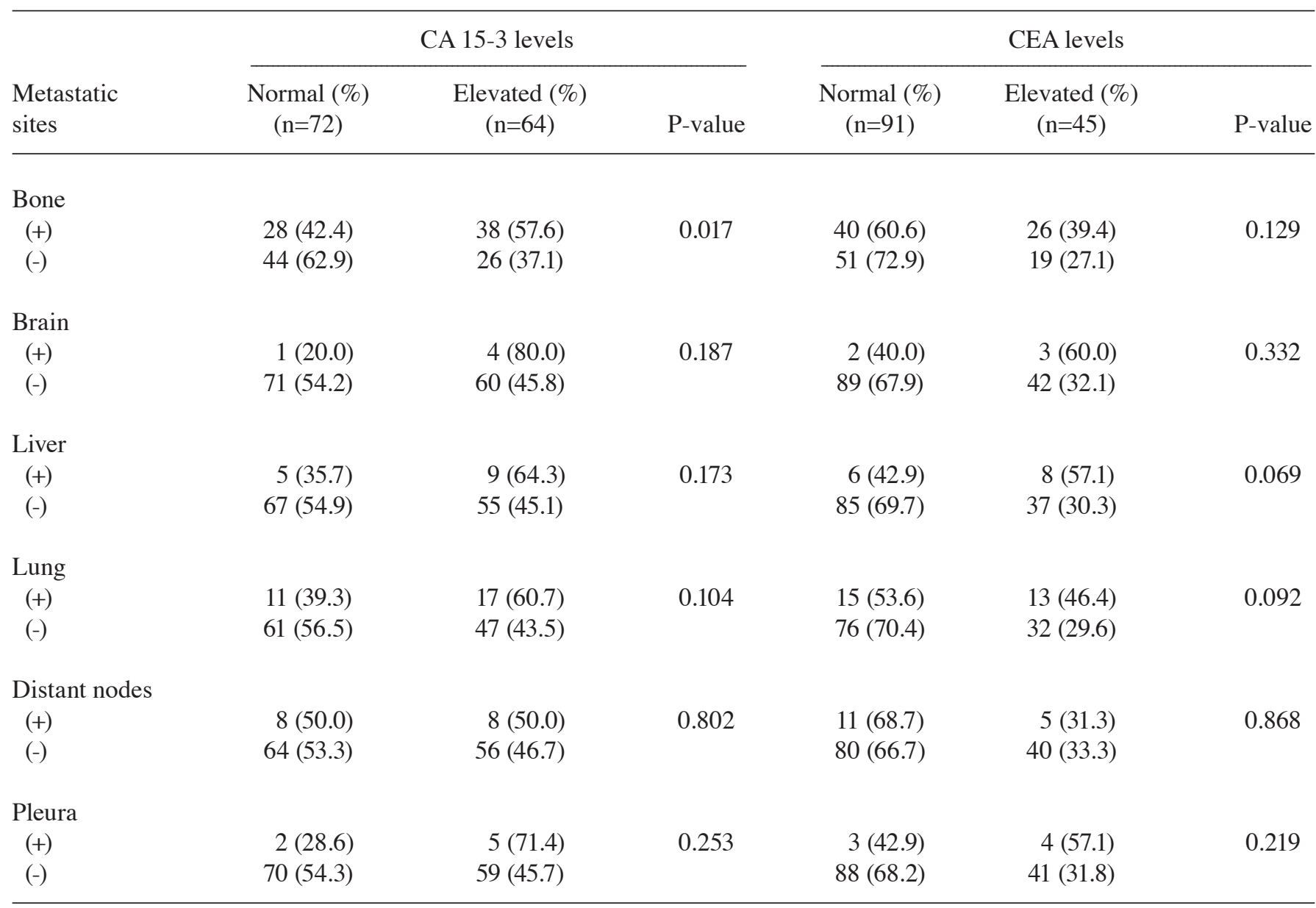

CA 15-3, cancer antigen 15-3; CEA, carcinoembryonic antigen.

Table IV. Correlation between CA 15-3 and CEA elevation and number of metastatic sites.

\begin{tabular}{|c|c|c|c|c|c|c|}
\hline \multirow{2}{*}{$\begin{array}{l}\text { No. of } \\
\text { metastatic } \\
\text { sites }\end{array}$} & \multicolumn{3}{|c|}{ CA 15-3 levels } & \multicolumn{3}{|c|}{ CEA levels } \\
\hline & $\begin{array}{c}\text { Normal }(\%) \\
\quad(n=121)\end{array}$ & $\begin{array}{l}\text { Elevated }(\%) \\
\quad(n=163)\end{array}$ & P-value & $\begin{array}{l}\text { Normal }(\%) \\
\quad(n=187)\end{array}$ & $\begin{array}{l}\text { Elevated }(\%) \\
\quad(n=97)\end{array}$ & P-value \\
\hline Single & $72(52.9)$ & $64(47.1)$ & 0.001 & $91(66.9)$ & $45(33.1)$ & 0.716 \\
\hline Multiple & $49(33.1)$ & 99 (66.9) & & 96 (64.9) & $52(35.1)$ & \\
\hline
\end{tabular}

CA 15-3, cancer antigen 15-3; CEA, carcinoembryonic antigen.

of recurrences prior to clinical or radiological evidence of disease, with a lead time of 2-18 months. The combined measurement of both serum markers allows early diagnosis of metastases in up to $60-80 \%$ of breast cancer patients $(12,13)$. It was previously demonstrated that CA 15-3 and CEA levels are elevated in $\sim 80$ and $40 \%$, respectively, of patients diagnosed with MBC $(14,15)$. In the present study, elevated CA 15-3 and CEA levels at initial diagnosis of recurrence were identified in $163(57.4 \%)$ and $97(34.2 \%)$ of the 284 patients, respectively, in accordance with previous findings.

Previous studies reported an association between elevated CA 15-3 levels and ER positivity, namely that the incidence of increased CA 15-3 levels was higher in patients with ER-positive primary tumors compared to those with ER-negative tumors $(9,16)$. Our results indicated that CA 15-3 and CEA elevation at initial diagnosis of $\mathrm{MBC}$ were associated with breast cancer molecular subtypes $(\mathrm{P}<0.001$ and $\mathrm{P}=0.032$, respectively). Elevated CA 15-3 and CEA levels were most commonly observed in luminal subtypes, whereas the incidence of CA 15-3 and CEA elevation was lower in the HER2-enriched and TN subtypes. The reason for this difference has not been fully elucidated. According to a previous study (17), the expression patterns of luminal, HER2-enriched and basal-like tumors are closely associated with mature 
luminal, luminal progenitor and the basal stem/progenitor cells of normal breast tissue, respectively. Based on that finding, it is hypothesized that less differentiated subtypes lack certain circulating antigens. Luminal subtypes are characterized by a high expression of hormone receptor (HR)-related genes, whereas HER2-enriched or basal-like subtypes exhibit a low expression of HR-related genes (18), indicating an association between CA 15-3 and CEA elevation and HR expression. In addition, a greater proportion of patients with preoperatively elevated tumor marker levels exhibited a more prominent elevation of tumor markers at recurrence compared to those without preoperatively elevated marker levels (19). The measurement of serum tumor markers is relatively easy and cost-effective. The incidence of the increase in CA 15-3 and CEA levels according to breast cancer molecular subtype may be useful in clinical practice.

As regards the correlation between marker levels and metastatic site(s), the results thus far have been conflicting: Tampellini et al (16) reported that increased CA 15-3 levels were found more frequently in patients with liver metastases and in those with pleural effusion. Yerushalmi et al (20) did not identify significant differences in CA 15-3 and CEA levels between different sites of metastasis, whereas al-Jarallah et al (21) reported that the highest proportions of elevated CA 15-3 levels were found in patients with bone metastases and the highest proportions of elevated CEA levels in patients with lung, bone or liver metastases. Our study demonstrated that the elevation CA 15-3 at recurrence was correlated with bone metastasis $(\mathrm{P}=0.017)$, whereas an elevated CEA level was observed regardless of metastatic site ( $P>0.05)$. Further studies, investigating the effect of metastatic site on CA 15-3 and CEA elevation, are required. Increased CA 15-3 was significantly more common in patients with $\geq 2$ metastatic sites compared to $\mathrm{MBC}$ with a single metastasis, in agreement with previously reported findings $(19,22)$. The significantly elevated CA 15-3 levels observed in cases with multiple metastases may reflect tumor burden.

In conclusion, CA 15-3 and CEA level elevation at initial diagnosis of recurrence were found to be associated with breast cancer molecular subtype. These serum tumor markers are frequently increased in the luminal subtypes of breast cancer. In addition, an elevated CA 15-3 level was correlated with bone metastasis, whereas elevated CEA was observed regardless of metastatic site. The elevation in CA 15-3 levels also differed according to the number of metastatic sites.

\section{References}

1. Li J, Zhang BN, Fan JH, et al: A nation-wide multicenter 10-year (1999-2008) retrospective clinical epidemiological study of female breast cancer in China. BMC Cancer 11: 364, 2011.

2. Early Breast Cancer Trialists' Collaborative Group (EBCTCG): Effects of chemotherapy and hormonal therapy for early breast cancer on recurrence and 15-year survival: an overview of the randomised trials. Lancet 365: 1687-1717, 2005.

3. Sayer HG, Kath R, Kliche KO and Höffken K: Premenopausal breast cancer: chemotherapy and endocrine therapy. Drugs 62: 2025-2038, 2002.
4. de Bono JS, Tolcher AW and Rowinsky EK: The future of cytotoxic therapy: selective cytotoxicity based on biology is the key. Breast Cancer Res 5: 154-159, 2003.

5. Harris L, Fritsche H, Mennel R, et al: American Society of Clinical Oncology 2007 update of recommendations for the use of tumor markers in breast cancer. J Clin Oncol 25: 5287-5312, 2007.

6. Park BW, Oh JW, Kim JH, et al: Preoperative CA 15-3 and CEA serum levels as predictor for breast cancer outcomes. Ann Oncol 19: 675-681, 2008.

7. Lee JS, Park S, Park JM, Cho JH, Kim SI and Park BW: Elevated levels of preoperative CA 15-3 and CEA serum levels have independently poor prognostic significance in breast cancer. Ann Oncol 24: 1225-1231, 2013.

8. Nicolini A, Tartarelli G, Carpi A, et al: Intensive post-operative follow-up of breast cancer patients with tumor markers: CEA, TPA or CA15.3 vs MCA and MCA-CA15.3 vs CEA-TPA-CA15.3 panel in the early detection of distant metastases. BMC Cancer 6: 269, 2006.

9. Pedersen AC, Sørensen PD, Jacobsen EH, Madsen JS and Brandslund I: Sensitivity of CA 15-3, CEA and serum HER2 in the early detection of recurrence of breast cancer. Clin Chem Lab Med 51: 1511-1519, 2013.

10. Yonemori K, Katsumata N, Noda A, et al: Development and verification of a prediction model using serum tumor markers to predict the response to chemotherapy of patients with metastatic or recurrent breast cancer. J Cancer Res Clin Oncol 134: 1199-1206, 2008

11. Hashimoto K, Yonemori K, Katsumata N, et al: Prediction of progressive disease using tumor markers in metastatic breast cancer patients without target lesions in first-line chemotherapy. Ann Oncol 21: 2195-2200, 2010.

12. Molina R, Barak V, van Dalen A, et al: Tumor markers in breast cancer - European Groupon Tumor Markers recommendations. Tumor Biol 26: 281-293, 2005.

13. Jäger W, Eibner K, Löffler B, Gleixner S and Krämer S: Serial CEA and CA 15-3 measurements during follow-up of breast cancer patients. Anticancer Res 20: 5179-5182, 2000.

14. Molina R, Jo J, Filella X, et al: c-erbB-2 oncoprotein, CEA, and CA $15-3$ in patients with breast cancer: prognostic value. Breast Cancer Res Treat 51: 109-119, 1998.

15. Lauro S, Trasatti L, Bordin F, et al: Comparison of CEA, MCA, CA 15-3 and CA 27-29 in follow-up and monitoring therapeutic response in breast cancer patients. Anticancer Res 19: 3511-3515, 1999.

16. Tampellini M, Berruti A, Gorzegno G, et al: Independent factors predict supranormal CA 15-3 serum levels in advanced breast cancer patients at first disease relapse. Tumor Biol 22: 367-373, 2001.

17. Lim E, Vaillant F, Wu D, et al: Aberrant luminal progenitors as the candidate target population for basal tumor development in BRCA1 mutation carriers. Nat Med 15: 907-913, 2009.

18. Cancer Genome Atlas Network: Comprehensive molecular portraits of human breast tumor. Nature 490: 61-70, 2012.

19. Lee JS, Park S, Park JM, Cho JH, Kim SI and Park BW: Elevated levels of serum tumor markers CA 15-3 and CEA are prognostic factors for diagnosis of metastatic breast cancers. Breast Cancer Res Treat 141: 477-484, 2013.

20. Yerushalmi R, Tyldesley S, Kennecke H, et al: Tumor markers in metastatic breast cancer subtypes: frequency of elevation and correlation with outcome. Ann Oncol 23: 338-345, 2012.

21. al-Jarallah MA, Behbehani AE, el-Nass SA, et al: Serum CA-15.3 and CEA patterns in postsurgical follow-up, and in monitoring clinical course of metastatic cancer in patients with breast carcinoma. Eur J Surg Oncol 19: 74-79, 1993.

22. Bidard FC, Hajage D, Bachelot T, et al: Assessment of circulating tumor cells and serum markers for progression-free survival prediction in metastatic breast cancer: a prospective observational study. Breast Cancer Res 14: R29, 2012. 